MS12-P04

\section{Identifying amyloid and partially amyloid structures from the protein data bank}

Vince Grolmusz ${ }^{1}$, Kristóf Takács ${ }^{1}$, Bálint Varga ${ }^{1}$

1. Eötvös University, PIT Bioinformatics Group, Budapest, Hungary email: grolmusz@pitgroup.org

The Protein Data Bank (PDB) contains 135000 entries today. From these, relatively few amyloid structures can be identified, since amyloids are insoluble in water. Therefore, mostly solid state NMR-recorded amyloid structures are deposited in the PDB. Based on the geometric analysis of these deposited structures we have prepared an automatically updated webserver, which generates the list of the deposited amyloid structures, and, additionally, those X-ray crystallography identifyid globular protein entries, which have amyloid-like substructures of a given size and characteristics. We have found that applying only the properly chosen geometric conditions, it is possible to identify the deposited amyloid structures, and a number of globular proteins with amyloid-like substructures. We have analyzed these globular proteins and have found that most of them are known to form amyloids more easily than many other globular proteins. Our results relate to the method of [1], who have applied a hybrid textual-search and geometric approach for finding amyloids in the PDB.

If one intends to identify a subset of the PDB for some applications, the identification algorithm needs to be re-run periodically, since in 2017 , on the average, every day 30 new entries were deposited in the data bank. Our webserver is updated regularly and automatically, and the identified amyloid- and partial amyloid structures can be viewed online or their list can be downloaded from the site https:// pitgroup.org/amyloid.

References:

1, Stanković, I. et al. (2017): Construction of Amyloid PDB Files Database. Transactions on Internet Research. 13 (1): 47-51.

Keywords: PDB, amyloids, webserver
MS12-P05

\section{Coordination of structural bioinformatics activities across Europe}

Bohdan Schneider ${ }^{1}$, Christine Orengo ${ }^{2}$, Torsten Schwede ${ }^{3}$, Joel L. Sussman $^{4}$, Janet M. Thornton ${ }^{5}$, Sameer Velankar ${ }^{5}$, Shoshana Wodak ${ }^{6}$

1. Laboratory of Biomolecular Recognition, Institute of Biotechnology of the Czech Academy of Sciences, Vestec, Czech Republic

2. University College London, London, United Kingdom

3. Biozentrum, University of Basel, Basel, Switzerland

4. Dept. of Structural Biology, Weizmann Institute of Science, Rehovot, Israel

5, European Molecular Biology Laboratory, European Bioinformatics Institutes, Hinxton, United Kingdom 6. VIB-VUB Center for Structural Biology, Brussels, Belgium email: bohdan.schneider@gmail.com

Structural bioinformatics has a broad impact across the life sciences and provides tools to archive, present, analyse, annotate, and predict molecular structures. The science of structural bioinformatics is traditionally very strong in Europe offering many software tools, methodologies, and databases, as well as community-wide prediction challenges. Its applications cover research activities from structural biology to drug discovery and personalized medicine that are all well represented within the national ELIXIR nodes. We believe that the coordination of the many concurrent activities will help to unite the voice of structural bioinformaticians in Europe and thus maximize the impact of structural bioinformatics to the benefit of all researchers in the field and the scientific community at large.

We therefore propose the establishment of an ELIXIR Community in Structural Bioinformatics to represent the broad group of activities in this field. We suggest calling it 3DBioInfo. We volunteer to act as an interim executive committee to organise a kick-off workshop for this Community, which would take place in the Fall of 2018. We will also establish an interim Steering Committee with a structural bioinformatics representative from each ELIXIR node to make sure we capture the interests and activities in each country. Researchers at the kick-off workshop will formulate the goals of the 3DBioInfo Community and collect ideas for Community activities to form the basis of a formal application to the ELIXIR Heads of Nodes.

Keywords: bioinformatics, molecular structure, ELIXIR 